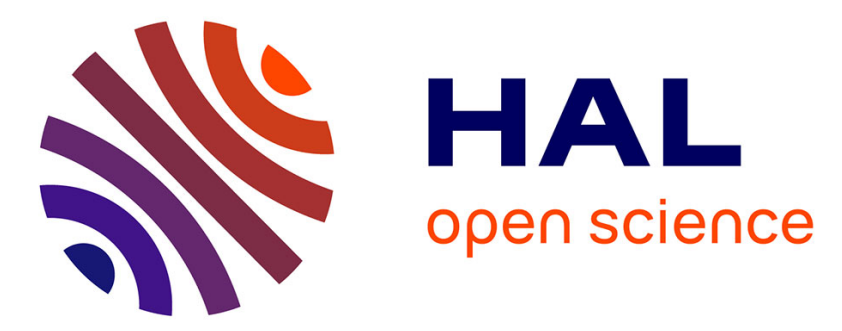

\title{
Pickett angles and Cremer-Pople coordinates as collective variables for the enhanced sampling of six-membered ring conformations.
}

\author{
Marcello Sega, Emmanuel Autieri, Francesco Pederiva
}

\section{- To cite this version:}

Marcello Sega, Emmanuel Autieri, Francesco Pederiva. Pickett angles and Cremer-Pople coordinates as collective variables for the enhanced sampling of six-membered ring conformations.. Molecular Physics, 2010, pp.1. 10.1080/00268976.2010.522208 . hal-00640226

\section{HAL Id: hal-00640226 \\ https://hal.science/hal-00640226}

Submitted on 11 Nov 2011

HAL is a multi-disciplinary open access archive for the deposit and dissemination of scientific research documents, whether they are published or not. The documents may come from teaching and research institutions in France or abroad, or from public or private research centers.
L'archive ouverte pluridisciplinaire HAL, est destinée au dépôt et à la diffusion de documents scientifiques de niveau recherche, publiés ou non, émanant des établissements d'enseignement et de recherche français ou étrangers, des laboratoires publics ou privés. 


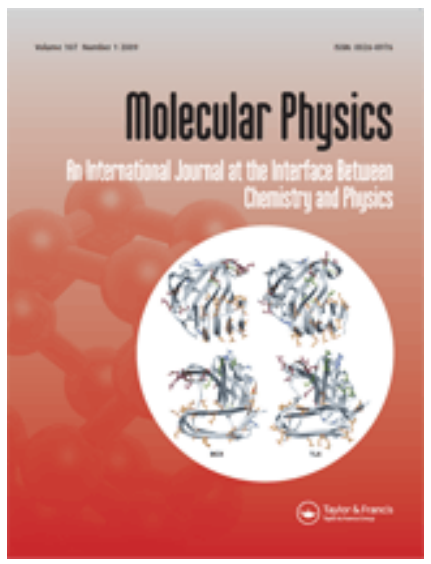

\section{Pickett angles and Cremer-Pople coordinates as collective variables for the enhanced sampling of six-membered ring conformations.}

\begin{tabular}{|r|l|}
\hline Journal: & Molecular Physics \\
\hline Manuscript ID: & TMPH-2010-0311 \\
\hline Manuscript Type: & $\begin{array}{l}\text { Special Issue Paper -EIGHTH LIBLICE CONFERENCE on the } \\
\text { Statistical Mechanics of Liquids }\end{array}$ \\
\hline Author: & 30 -Jul-2010 \\
\hline Complete List of Authors: & $\begin{array}{l}\text { Sega, Marcello; University of Trento, Dept. of Physics; INFN } \\
\text { Autieri, Emmanuel; University of Trento, Dept. of Physics; INFN } \\
\text { Pederiva, Francesco; University of Trento, Dept. of Physics; INFN }\end{array}$ \\
\hline Keywords: & Puckering, Sugars, Ring conformers, Free energy \\
\hline \multicolumn{2}{|c|}{} \\
\hline $\begin{array}{l}\text { Note: The following files were submitted by the author for peer review, but cannot be converted } \\
\text { to PDF. You must view these files (e.g. movies) online. }\end{array}$ \\
\hline \begin{tabular}{l} 
molphys.zip \\
\hline
\end{tabular}
\end{tabular}

\section{SCHOLARONE \\ Manuscripts}




\title{
RESEARCH ARTICLE
}

\author{
Pickett angles and Cremer-Pople coordinates as collective \\ variables for the enhanced sampling of six-membered ring \\ conformations.
}

\author{
M. Sega ${ }^{a *}$, E. Autieri ${ }^{a}$, and F. Pederiva ${ }^{a}$ \\ ${ }^{a}$ Department of Physics and I.N.F.N., University of Trento \\ (Received 00 Month 200x; final version received 00 Month 200x)
}

\begin{abstract}
Although completely equivalent for the description of puckered ring conformers, the two popular coordinates sets of Strauss-Pickett dihedral angles and Cremer-Pople spherical coordinates are shown to have contrasting features when employed as collective variables in free energy calculations with accelerated sampling techniques. Results from a $100 \mathrm{~ns}$ molecular dynamics simulation at conformational equilibrium and from combined metadynamics/umbrella sampling calculations of glucose are exploited to elucidate these differences.
\end{abstract}

Keywords: Puckering, Sugars, Ring conformers, Free energy

\section{Introduction}

Cyclic structures are ubiquitous in nature, and appear mostly in non-planar, puckered conformations. The problem of an intuitive description of puckered ring conformers dates back to the work of Sachse[1], but it is only in 1975 that an exhaustive, mathematically correct description of a general $N$-membered ring puckering has been proposed[2]. The description of Cremer and Pople (CP) has from time to time been questioned because of some conflicts with the standard stereochemical interpretation of puckering[3], of its not straightforward way to determine atomic positions from puckering parameters[4-6], and for some difficulties in comparing with NMR experimental results[5, 7]. Nevertheless, the CP approach has become the most widely employed way to describe puckered conformers. The calculation of puckering free energy landscapes, and the determination of conformer populations has been the subject of a series of recent computer simulation investigations [8-12], apparently stimulated by the possibilities offered by recent developments in accelerated dynamics methods. Among those works, Strauss-Pickett (SP) dihedrals[1315] have been also employed, as an alternative to $\mathrm{CP}$ variables, to perform the exploration of the puckering free energy space.

In this work we address the advantages and disadvantages related to the choice of $\mathrm{CP}$ and SP coordinates, from the perspective of who is interested in computing the population of puckered conformers by means of computer simulations, in particular with the help of accelerated dynamics methods.

\footnotetext{
*Corresponding author. Email:sega@science.unitn.it
} 


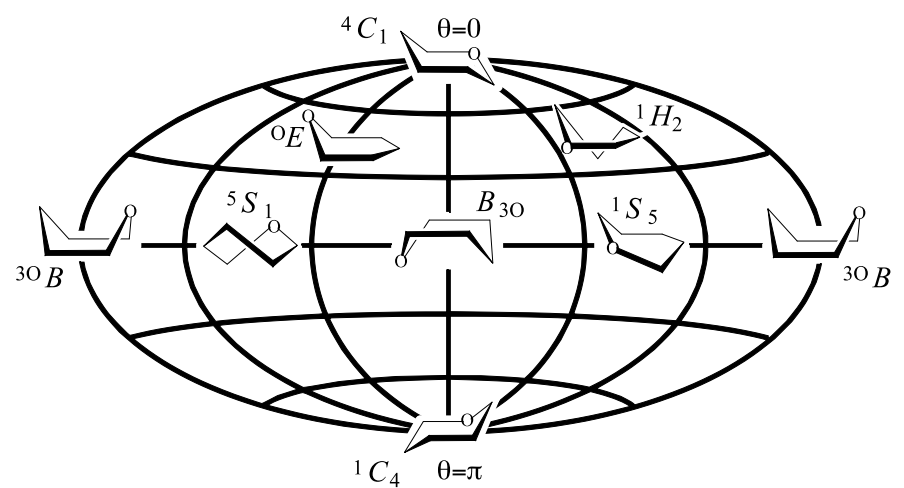

(a) The Hammer-Aitoff equal-area projection[23] of the CP conformational globe. Conventional names and ring structures of some hexopyranose conformers are shown.

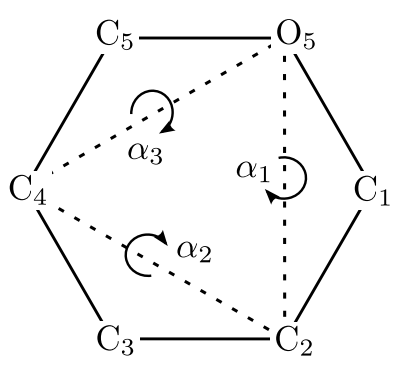

(b) Definition[15] of the out-ofplane dihedral angles (SP puckering representation).

Figure 1. Location of ring conformers on the CP globe and definition of SP dihedrals.

\section{Puckering Coordinates and accelerated sampling methods}

\subsection{Puckering coordinates}

The description of ring conformation can be either qualitative (that is, by assigning conventional names to special ring conformers) or quantitative (employing a parametric measure of the deviation from a reference shape). While IUPAC indications $[16,17]$ realize the first of these two descriptions, no agreement on a standard quantitative measure has been reached so far. The first attempt to provide a set of parameters to describe ring conformers has been the observation of Kilpatric and coworkers[18, 19], who employed for the first time the concept of a puckering amplitude and a pseudorotation angle to describe the puckered conformers of five-membered rings. A more systematic study of this problem came with the group theory analysis of Pickett and Strauss[20,21] and eventually with the first general definition of ring puckering coordinates of Cremer and Pople[2, 6]. Both SP and CP approaches describe the conformation of a $N$-membered ring with $N-3$ parameters, expressed in terms of the elevations of the ring atoms from a reference plane (the mean ring plane for $\mathrm{CP}$ coordinates, and the planar ring conformation for SP coordinates). These two approaches, proven to be equivalent[22], represented the first mathematically consistent and quantitative measure of ring conformers for the puckering problem.

Cremer-Pople coordinates[2] are expressed in terms of the atomic elevations $z_{j}$ from the mean ring plane defined using the Cartesian coordinates of all ring atoms. In the case of six-membered rings the elevations from the mean plane,

$$
z_{j}=\frac{1}{\sqrt{3}} q_{2} \cos \left[\phi_{2}+\frac{2 \pi(j-1)}{3}\right]+\frac{1}{\sqrt{6}} q_{3}(-1)^{j-1}
$$

depend on three puckering parameters, $q_{2}, \phi_{2}$ and $q_{3}$. These coordinates are usually re-written as a set of spherical coordinates $(Q, \theta, \phi)$, so that

$$
\left\{\begin{array}{l}
q_{2} \cos \phi_{2}=Q \sin \theta \cos \phi \\
q_{2} \sin \phi_{2}=Q \sin \theta \sin \phi \\
q_{3}=Q \cos \theta
\end{array}\right.
$$

In this case the whole conformational space (see Fig.1a) is spanned by $Q>0$, $\theta \in\left[0,180^{\circ}\right]$ and $\phi \in\left[0,360^{\circ}\right)$. The radial coordinate, $Q$, also called total puckering 
Table 1. Location of carbohydrate conformations in the SP dihedral space ${ }^{\text {a }}$.

\begin{tabular}{lrrr}
\hline Conformer & $\alpha_{1}(\mathrm{deg})$ & $\alpha_{2}(\mathrm{deg})$ & $\alpha_{3}(\mathrm{deg})$ \\
\hline${ }^{4} C_{1}$ & -35 & -35 & -35 \\
${ }^{1} C_{4}$ & 35 & 35 & 35 \\
${ }^{B} 3$ & 30 & -60 & 30 \\
$B_{25}$ & 30 & 30 & -60 \\
${ }^{B}{ }_{14}$ & -60 & 30 & 30 \\
${ }^{3}{ }_{B}$ & -30 & 60 & -30 \\
${ }^{25} B$ & -30 & -30 & 60 \\
${ }^{14} B$ & 60 & -30 & -30 \\
${ }^{1} S_{3}$ & 60 & -60 & 0 \\
${ }^{1} S_{5}$ & 60 & 0 & -60 \\
${ }^{3} S_{5}$ & 0 & 60 & -60 \\
${ }^{3} S_{1}$ & -60 & 60 & 0 \\
${ }^{5} S_{1}$ & -60 & 0 & 60 \\
${ }^{5} S_{3}$ & 0 & -60 & 60 \\
\hline
\end{tabular}

${ }^{\mathrm{a}}$ From Rao et al.[15].

amplitude, is related to the elevations by the simple relation $Q^{2} \equiv \sum_{j=1}^{N} z_{j}^{2}=$ $\sum_{m} q_{m}^{2}$. Chair conformers are located on the north and south pole of the puckering sphere, while other conformers like boats, and skew boats are found along the pseudorotational path at the equatorial line.

The analysis of Pickett and Strauss, on the contrary, relied on the definition of the elevation $Z_{j}$ from a planar ring structure, and used group theory to identify the three parameters that can describe the puckering of a six-membered ring. Three out-of-plane dihedrals, $\alpha_{1}, \alpha_{2}, \alpha_{3}$ (see Fig.1) can be defined to be the three puckering parameters. For sugar rings, these dihedral angles are defined as: $\alpha_{1}=\mathrm{C}_{4^{-}}$ $\mathrm{O}_{5}-\mathrm{C}_{2}-\mathrm{C}_{1}-180^{\circ}, \alpha_{2}=\mathrm{O}_{5}-\mathrm{C}_{2}-\mathrm{C}_{4}-\mathrm{C}_{3}-180^{\circ}, \alpha_{3}=\mathrm{C}_{2}-\mathrm{C}_{4}-\mathrm{O}_{5}-\mathrm{C}_{5}-180^{\circ}$, where the standard notation for ring atoms ( $\mathrm{C} 1$ being the anomeric carbon) has been used. The SP description differs form the $\mathrm{CP}$ one only in the usage of the $Z=0$ plane rather than the $z=0$ mean plane, while the other the principles are the same[22]. These angles measure the orientation of tree "flaps" involving different atoms that can be identified on a six-membered rings (see Fig.1b). Ring conformers are usually located in the $\left[-90^{\circ}, 90^{\circ}\right] \times\left[-90^{\circ}, 90^{\circ}\right] \times\left[-90^{\circ}, 90^{\circ}\right]$ sub-volume. Common conformers for carbohydrates are listed in Tab.1. The three out-of-plane angles $\alpha_{i}$ can also be rewritten $[20]$ as

$$
\alpha_{i}=R[\cos \Theta+2 \sin \Theta(4 \pi i / 3-2 \pi / 3+\Phi)],
$$

where again a spherical set of coordinates $(R, \Theta, \Phi)$ is employed to describe the conformational phase space. It is worth remarking that this spherical coordinate set is different from the $(Q, \theta, \phi)$ one, again because the two approaches differs in the definition of the reference plane.

\subsection{Accelerated sampling methods}

A large number of accelerated dynamics methods, such as metadynamics[24], adaptive umbrella sampling[25], or local elevation[26] to cite but a few, have been proposed in the last decade to address the problem of investigating rare events (discussions regarding different enhanced sampling techniques can be found in Refs. [10, 27]). The introduction of a time dependent biasing force which enforces the exploration of the complete phase space, at the price of loosing the physical meaning of microscopic trajectories, is the common trait of accelerated dynamics methods. The set of collective variables on which the biasing force is acting, has 
Here $U_{\text {bias }}$ is the biasing potential $k_{\mathrm{B}}$ is Boltzmann constant, $T$ is the temperature, $\rho_{\text {bias }}(s)$ is the probability density of finding the system at $s$ during the umbrella sampling phase and $\langle\ldots\rangle_{b}$ denotes an average performed over the biased ensemble.

\subsection{Simulation details}

In this work we present results from different simulations (molecular dynamics, combined metadynamics/umbrella sampling in presence and absence of solvent, using $\mathrm{CP}$ or SP coordinates) involving $\beta$-D-glucose. In all simulations glucose molecules have been modeled using the GROMOs 45a4 force field[31], while the SPC model[32] has been employed for water molecules. Even though newer versions of the gromos force field provide a better description of the puckering properties of sugars[30, 33], the $45 \mathrm{a} 4$ force field has been chosen for the present work because it allowed to estimate by standard molecular dynamics the equilibrium population of the chair conformers of glucose, therefore enabling us to check in a direct way the predictions of the coupled metadyamics/umbrella sampling approach with CP collective variables.

All simulations has been performed at a constant temperature of $300 \mathrm{~K}$, employing a Nosè-Hoover thermostat with time constant of 0.1 ps for the simulation in solution and a Langevin thermostat with time constant of $0.1 \mathrm{ps}$ for the simulation in vacuum. In addition, for simulations in solution, the edges of the simulation box have been rescaled by means of the Parrinello-Rahman algorithm[34] with a time constant of 1 ps to keep a constant pressure of 1 atm. In this case, long range correction for dispersion forces have been applied. Bond lengths have been always kept constant at their equilibrium value by means of the SHAKE algorithm[35], us- 
ing a relative tolerance of $0.1 \%$. The simulation performed will be conventionally called simulation I-III. For simulations II and III, cutoffs of $1 \mathrm{~nm}$ have been applied to Lennard-Jones and electrostatic interactions, while for simulation I the cutoff length was $1.3 \mathrm{~nm}$. In addition, electrostatic interactions have been screened by using a dielectric constant of 80 , for the simulations in vacuum.

Simulation $I$ is a $150 \mathrm{~ns}$ long molecular dynamics simulation (with an integration timestep of $2 \mathrm{fs}$ ) of 512 glucose molecules in 25512 solvent molecules of solvent. This represent a big enough statistical sample to observe conformational changes, allowing to check the prediction of the metadynamics/umbrella sampling approach by estimating equilibrium population of chair conformers and, in addition, transition rates. Configurations along the trajectory were sampled every $10 \mathrm{ps}$ and saved to disk for off-line analysis.

Simulation II is a metadynamics of a single glucose molecule in vacuum, performed by applying the bias potential on the two angular CP coordinates. During the metadynamics run, about $10^{5}$ Gaussians were placed each every $0.1 \mathrm{ps}$, with a height of $0.12 \mathrm{~kJ} / \mathrm{mol}$, and a width of $0.05 \mathrm{rad}$ in each angular direction. The free energy profile has been generated by calculating the value of the biasing potential on a $60 \times 60$ grid in the $(\theta, \phi)$ space.

Simulation III is a metadynamics of a single glucose molecule in vacuum, performed by applying the bias potential on the three SP dihedrals. The number, height and frequency of Gaussians placed are the same as in simulation II, while their width was set to $0.2 \mathrm{rad}$ in each of the three angular direction. The free energy profile has been generated by calculating the value of the biasing potential on a $60 \times 60 \times 60$ grid in the $\left(\alpha_{1}, \alpha_{2}, \alpha_{3}\right)$ space.

\section{3. $\mathrm{CP}$ and $\mathrm{SP}$ variables as reaction coordinates}

\subsection{The state-counting problem}

Even though CP and SP coordinates are equally fit to describe puckered conformers from a purely geometrical point of view, differences can arise due to the shape of the puckering free energy hypersurfaces. Usually, in six-membered rings the CP puckering amplitude $Q$ is not a slow degree of freedom, as it does not present metastabilities, and its free energy profile is such that conformers are generally populating only a thin spherical shell of the $\mathrm{CP}$ conformational globe[9]. It is therefore sufficient to introduce a biasing potential along the angular $\mathrm{CP}$ variables only, in order to get a proper exploration of the conformational phase space. Moreover, every coordinate system has to be complemented by a suitable assignment scheme for thermodynamic state, as geometrically different (in the sense of CP or $\mathrm{SP}$ coordinates) conformers located in the same free energy basin represent a single conformer in the thermodynamical sense.

Given the presence of only two variables, in case of $\mathrm{CP}$ variables visual inspection is sufficient to assign thermodynamic states, while quantitative schemes are required for SP variables. This can be easily seen by looking at the free energy profiles obtained from simulations II and III, presented in Fig.2 and 3, respectively. For simulation III, which employed SP dihedral angles, only some relevant cross sections of the free energy volume are presented, namely those at $\alpha_{3}=-60,-35,0,35$ and $60 \mathrm{deg}$. By looking at the free energy profiles presented, one can readily understand how different can be the task of identifying conformers location, free energy basins and barriers in the two coordinate systems.

In the LEUS investigation of Hünenberger and Hansen[10], the authors proposed three quantitative assignment schemes suitable for SP variables. Two of 


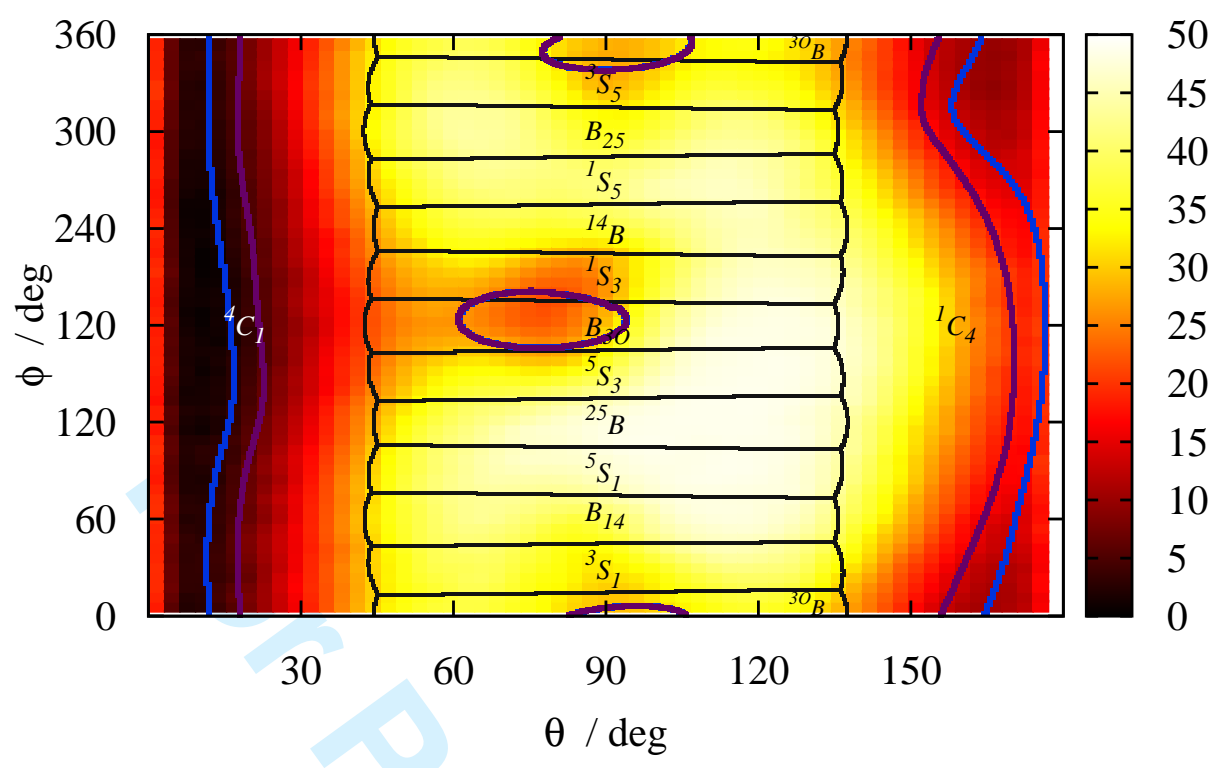

Figure 2. Free energy profile obtained from Simulation II. The superimposed solid lines defines the regions determined by the CD (black lines), projected onto the $(\theta, \phi)$ plane . The conventional symbols of conformers associated to the different regions of the CD scheme are also shown. Blue and violet solid lines represent the projections of the ellipsoids for the MS scheme using a 5 and $10 \mathrm{~kJ} / \mathrm{mol}$ threshold, respectively.

these schemes, namely the closest distance (CD) and angle deviation (AD) ones are solely geometrical, while the minimum shape one (MS) employs a mixed energetic / geometrical criterion.

In the CD scheme, a conformation identified by the triplet of SP dihedrals $\beta=$ $\left(\beta_{1}, \beta_{2}, \beta_{3}\right)$ is assigned to the $i$-th state $\alpha^{i}=\left(\alpha_{1}^{i}, \alpha_{2}^{i}, \alpha_{3}^{i}\right)$ if the quantity $d^{i}(\beta)=$ $1-\alpha^{i} \cdot \beta /\left(\left\|\alpha^{i}\right\|\|\beta\|\right)$ is smaller than that of other states $d^{j}(\beta)$ (See Tab.1 for the definition of the triplets defining different puckered states). At a difference with the CD partitioning scheme, in the MS one states are identified by ellipsoids enclosing a local minimum and all the nearby conformations within an energy interval of 5 $\mathrm{kJ} / \mathrm{mol}$ (the details are reported in [10]). Although the criterion is still partially geometrical, since it assumes an ellipsoidal shape of the basins, it makes use of the information from the free energy landscape, and is thus, among the three SP partitioning schemes, the closest to the visual inspection approach used with $\mathrm{CP}$ variables.

In the following we compare how three different schemes (visual inspection for CP coordinates; CD and MS for SP coordinates) partition the respective conformational space. It is therefore necessary to represent the thermodynamic states in a common framework. The natural way to do this is to project the volumes of states assigned in the SP space onto the CP plane. In Fig.2 it is possible to see the different sub-volumes of the SP space corresponding to the 14 states determined by the CD assignment, projected onto the $(\theta, \phi)$ plane. The 14 regions have been determined by reconstructing $[6]$ the atomic positions of an ideal conformer (i.e. using bond lengths and angles of an ideal cyclohexane molecule). Starting from the CP puckering angles taken from a $500 \times 500$ grid on the $(\theta, \phi)$ plane, SP dihedrals have eventually been calculated from the atomic coordinates, and the CD scheme has been applied (the computer code which computes atomic positions from CP parameters 


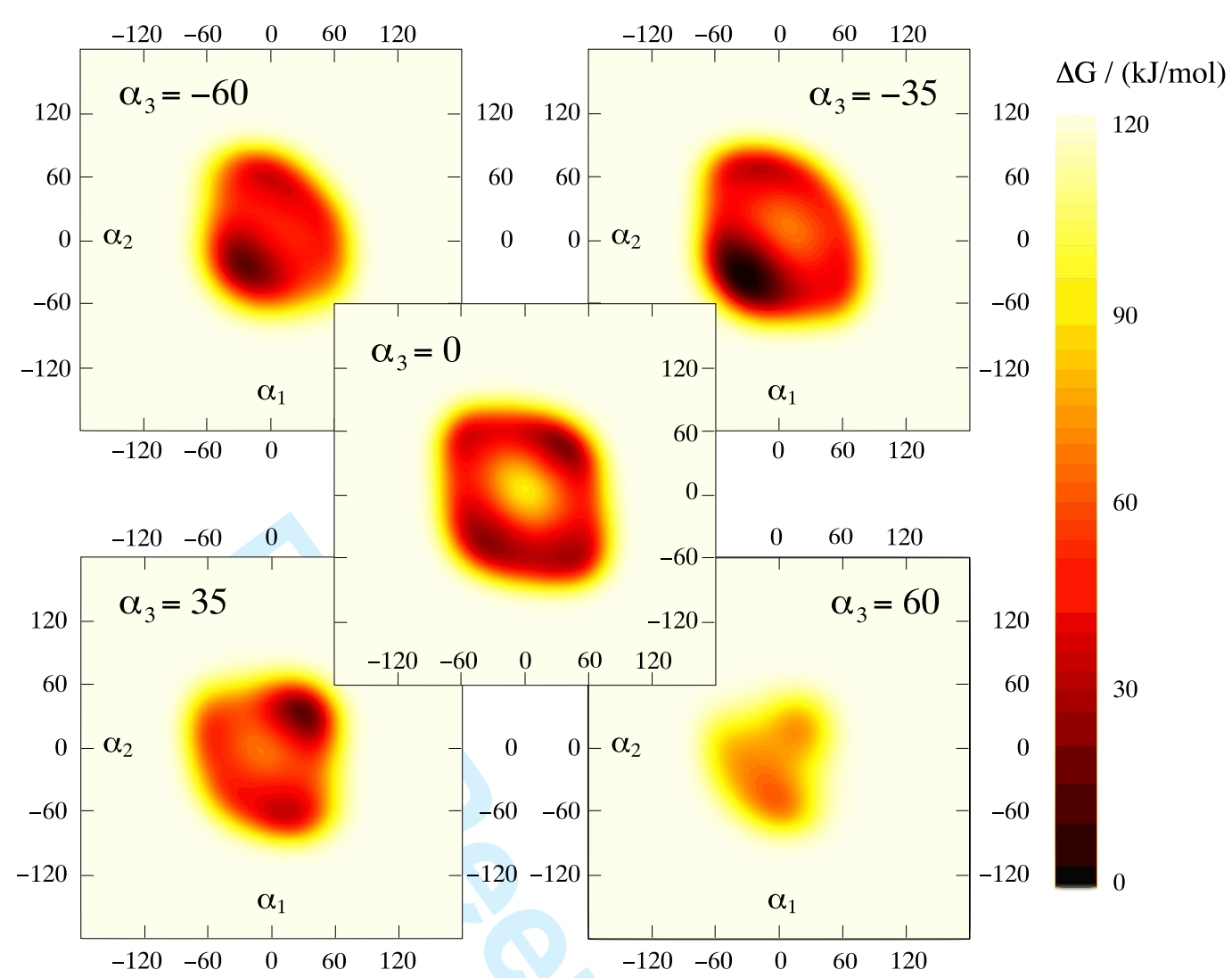

Figure 3. Cross sections (at $\alpha_{3}=-60,-35,0,35$ and $60 \mathrm{deg}$ ) of the puckering free energy profile obtained from Simulation III.

is made available at http://www.science.unitn.it/ sega/sugars.html.) In the background, the free energy profile of hydrated $\beta$-D-glucose obtained with the metadynamics/umbrella sampling approach[30] is shown. It is clearly seen that along the pseudorotational (equatorial) path, states determined by their thermally accessible conformational volume encompass more than one state defined by geometry considerations. This is the cases of the free energy basin around $\phi=0^{\circ}$ that covers a group of five geometrically different conformers (namely $B_{25},{ }^{O} S_{2},{ }^{3 O} B,{ }^{3} S_{1}, B_{14}$ ), or also the basin around $\phi=180^{\circ}$ that groups four different conformers (namely $\left.{ }^{2} S_{O}, B_{3 O},{ }^{1} S_{3},{ }^{14} B\right)$. This confirms what Hünenberger and Hansen noted in their work, namely, that purely geometrical criteria such as CD and AD do not allow to identify thermodynamic states (intended as free energy basins). The regions associated with the two chair conformers, at the north and south pole, respectively, seem on the contrary to capture quite nicely the features of the underlying free energy profile.

Using the same procedure employed for the CD scheme, we calculated the projection of the MS ellipsoids obtained from the SP free energy profile onto the $(\theta, \phi)$ plane, using two different threshold values of 5 and $10 \mathrm{~kJ} / \mathrm{mol}$, respectively. As it can be seen from Fig.2, the MS scheme is able to describe noticeably well the shape of the main four free energy basins, however the full extent of the basins is not covered, at least with the $5 \mathrm{~kJ} / \mathrm{mol}$ threshold. Notice that changing the threshold from 5 to $10 \mathrm{~kJ} / \mathrm{mol}$ affects only the assignment of chair conformer basins, but not the boat and skew ones, as only the latter basins are less deep than $5 \mathrm{~kJ} / \mathrm{mol}$.

To check quantitatively the CD and MS schemes for chairs, we compared the percentage of these conformers along the evolution of simulation I estimated using the CD, MS and visual inspection schemes. Regarding the visual inspection, in 


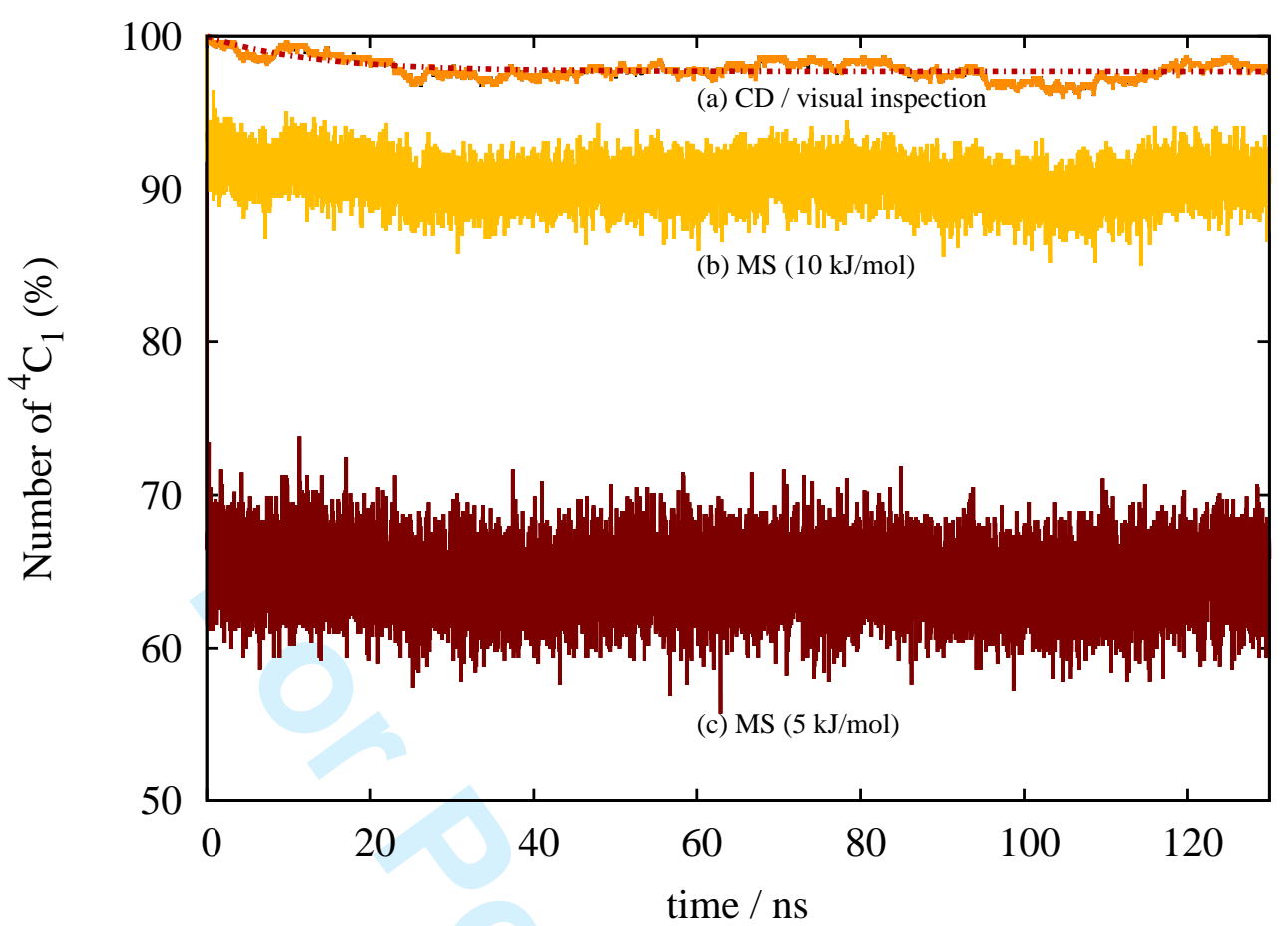

Figure 4. Time evolution of the population of ${ }^{4} C_{1}$ conformers in simulation I. Solid lines from top to bottom: (a) the CD scheme (almost coincident with the visual inspection scheme result); (b) the MS scheme with $10 \mathrm{~kJ} / \mathrm{mol}$ threshold and (c) the MS scheme with $5 \mathrm{~kJ} / \mathrm{mol}$ threshold. A fit to an exponential decay (dot-dashed line) for (a) is also presented.

particular, from the free energy profile reported in Fig.2 one can see that the location of the free energy barrier for the chair is very well approximated by the region $\theta<60$. We reported in Fig. 4, the time evolution of the number of chair conformer, computed using the two criteria.

The time evolution of population of chairs and inverted chairs $(\theta>120)$ in the simulation box is practically indistinguishable for the CD and visual inspection schemes, and amounts to a relative root mean square difference between the two methods of 0.02 and $0.03 \%$ for chairs and inverted chairs, respectively, proving SP dihedrals and the $\mathrm{CD}$ approach quite robust for the identification of chairs. The MS scheme shows an interesting behavior: when using the $5 \mathrm{~kJ} / \mathrm{mol}$ threshold, only roughly $60 \%$ of chairs (and inverted chairs) with respect to the visual inspection or CD schemes are observed, meaning a miscounting of states. However, since the measured populations are reduced by roughly the same ratio for both chairs and inverted chairs, the calculation of free energy difference using Boltzmann inversion formula gives a value of $10.0 \mathrm{~kJ} / \mathrm{mol}$, compatible with the other schemes $(9.2$ $\mathrm{kJ} / \mathrm{mol}$ ). This situation can be improved, as seen in Fig.4, by using a $10 \mathrm{~kJ} / \mathrm{mol}$ threshold, which enlarges the assigned basins of chairs. It has to be noted, however, that since the smaller basins of the boat and skew conformers do not change shape, the evaluation of their free energy difference will be indeed affected by the choice of the threshold.

From the results presented in Fig.4 one can extract some additional, interesting information. A fit to an exponential decay, which assumes a reaction kinetic of the first order, shows that the population of chairs reaches the equilibrium value of $97.7 \%$ with a rate of about $13 \mathrm{~ns}^{-1}$, showing still large oscillations on the $40 \mathrm{~ns}$ scale. The estimated population corresponds to a free energy of $9.3 \mathrm{~kJ} / \mathrm{mol}$, in good agreement with the estimate of $10 \mathrm{~kJ} / \mathrm{mol}$ previously obtained by means of 
combined metadynamics/umbrella sampling[30].

\subsection{Influence of the collective variable choice on convergence}

Apart from the practical difficulties in identifying conformers discussed so far, one could argue that much more time should be spent to fill the free energy profile with the biasing potential in the SP case, with respect to the $\mathrm{CP}$ one, for the simple reason that the SP space is three-dimensional, whereas the CP is only twodimensional. In general, indeed, the time spent to sample a space grows exponentially with the number of its dimensions. However, this is true only if the different subspaces have the same characteristics, from an energetic and geometrical point of view. Including, for example, the total puckering amplitude in a metadynamics investigation with $\mathrm{CP}$ variables, will not increase dramatically the amount of phase space accessible at a certain energy, which will still have the shape of a spherical shell. Simple dimensional arguments are therefore not sufficient to estimate how the efficiency in filling the free energy profile depends on the set of collective variables in use. One possible estimate can be obtained, for example, by checking how many depositions are needed in order to fill with Gaussians a given amount of the energetically accessible portion of the free energy profile, $\Delta G$, once the system has become ergodic.

Obviously, the portion filled by Gaussians for a given number of deposition will depend on the Gaussian height, but also on its width. Rescaling the number of depositions by the thermal energy in units of the Gaussians height is the obvious way to compare the outcome of two simulations that makes use of different heights. However, different coordinates requires in general the use of different values for the Gaussians width, but at a difference with the heights, they are in general not commensurable. In practice, a characteristic size always emerges naturally from the problem, and is the size of the smallest feature of interest in the free energy landscape. When setting a Gaussian width much smaller than this characteristic size, time will be spent without gathering important information on the free energy landscape, whereas if the width is too large, a metadynamics run will not be able to resolve properly the desired feature. In order to provide a meaningful comparison, the number of deposition have to be rescaled by the volume of a reference feature, in units of the width of the Gaussians. For the puckering free energy landscape of glucose one could choose the volume accessible within one unit of thermal energy, $k_{\mathrm{B}} T$ from the minimum of a given conformer like, for example, ${ }^{3 O} \mathrm{~B}$.

In Fig.5 we present the equivalent number of depositions (that is, normalized according to the procedure described above) needed to add a minimum number of points to each of the 14 states listed in Tab.1, identified using the CD scheme. The surface and volume of the ${ }^{3 O} B$ conformer basin are $0.4 \mathrm{rad}^{2}$ and $1.2 \times 10^{-3} \mathrm{rad}^{3}$, respectively. The tendency for both $\mathrm{CP}$ and $\mathrm{SP}$ cases is to reach a plateau value, which denotes an homogeneous filling of the free energy landscape. A fit to an exponential decay has also been performed, which gives an estimate plateau value of about 120 and 2300 equivalent Gaussians for the case of CP and SP variables, respectively. This result shows that using $\mathrm{CP}$ variables can lead to an tenfold increase in efficiency in filling the free energy landscape with respect to the SP dihedral angles, when using the same Gaussian height and an equivalent Gaussian width. 


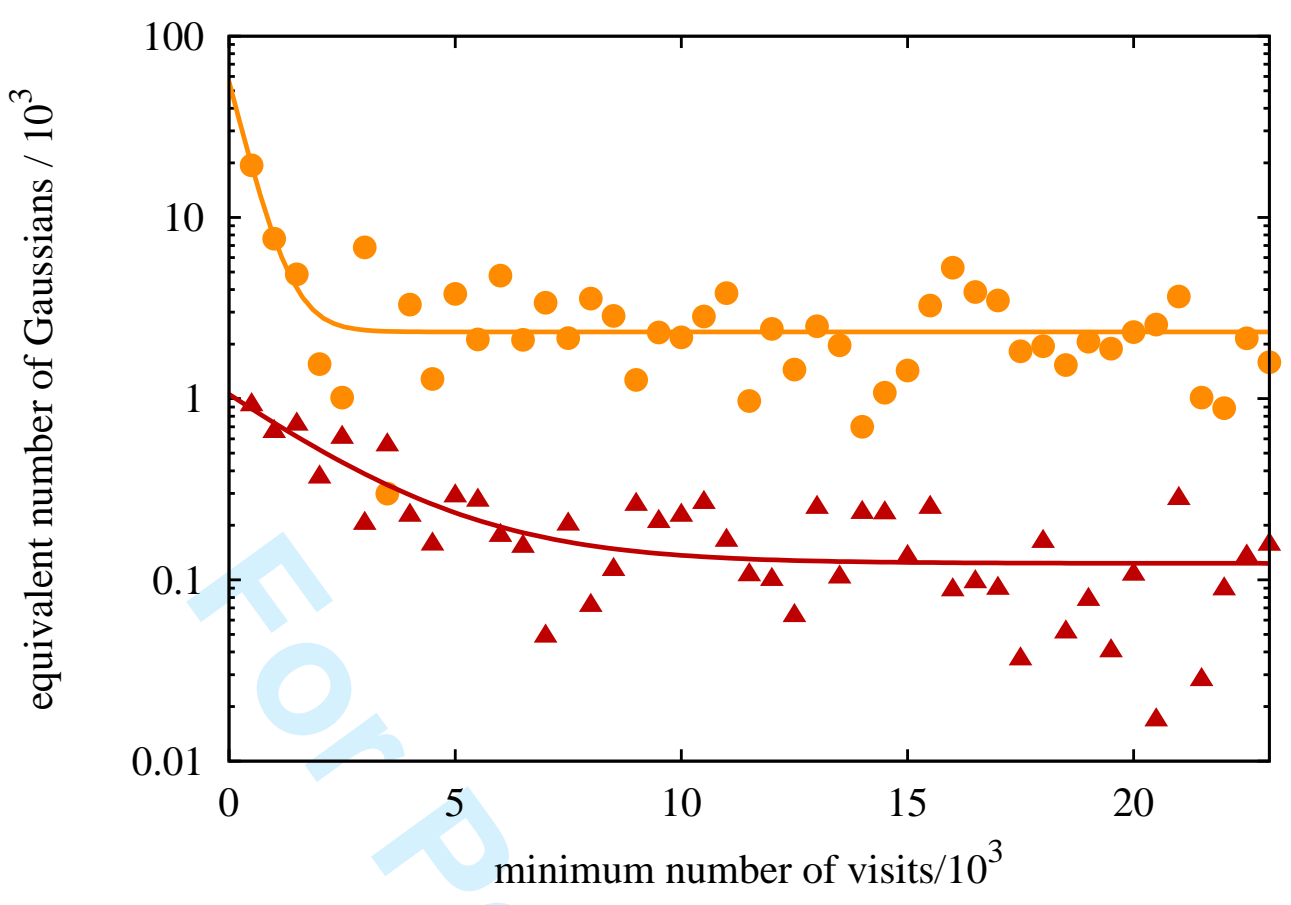

Figure 5. Efficiency of the CP and SP variables in filling the conformational free energy profile. Circles: CD scheme for SP dihedrals; Triangles: visual inspection criterion fo CP variables. The result of a fit to an exponential decay is also provided for each dataset.

\section{Conclusions}

Different equivalent descriptions, such as the Cremer-Pople and Strauss-Pickett ones, can be adopted to characterize puckered ring conformers. Their equivalence on the pure geometrical level does not correspond, however, to an equivalence on the practical level, when the puckering coordinate are employed as collective variables in accelerated dynamics methods to compute puckering free energy landscapes.

The need for only two (angular) puckering coordinates to sample in an ergodic way the conformational space in the Cremer-Pople approach is at the root of two main advantages with respect to the usage of Strauss-Pickett dihedrals. The first advantage is that energetically-separated conformational basins are readily identified in the CP scheme, whereas when employing the three SP dihedrals, one has to rely on some algorithmic partitioning of the conformational space. Two of the checked partitioning schemes, namely the closest distance and the minimum shape, are shown to be prone to a misleading interpretation of the thermally accessible conformers of sugar rings. The reason for the miscounting in the closest distance scheme is related to its purely geometrical nature, while in the minimum shape scheme the miscounting is related to the choice of the energy threshold. The second advantage is that employing CP coordinates rather than SP dihedrals leads to a tenfold decrease of the time needed to homogeneously fill the conformational space.

\section{Acknowledgements}

The authors thank P. Hünenberger and H. Hansen for fruitful discussions. Use of the HPC cluster Wiglaf at the Department of Physics of the University of Trento is acknowledged. The authors are member of the Interdisciplinary Laboratory of 
Computational Science (LISC).

\section{References}

[1]H. Sachse, Ber. Dtsch. Chem. Ges. 23, 1363 (1890).

[2]D. Cremer and J.A. Pople, J. Am. Chem. Soc. 97, 1354 (1975).

[3]N. Zefirov, V. Palyulin and E. Dashevskaya, J. Phys. Org. Chem. 3 (3), 147-158 (1990).

[4] A. Hill and P. Reilly, J. Chem. Inf. Model 47 (3), 1031-1035 (2007).

[5]A. Bérces, D. Whitfield and T. Nukada, Tetrahedron 57 (3), 477-491 (2001).

[6]D. Cremer, J. Phys. Chem. 94, 5502 (1990).

[7] C. Haasnoot, J. Am. Chem. Soc. 114 (3), 882-887 (1992).

[8]X. Biarnes, A. Ardevol, A. Planas, C. Rovira, A. Laio and M. Parrinello, J. Am. Chem. Soc. 129 (35), 10686-10693 (2007)

[9] M. Sega, E. Autieri and F. Pederiva, J. Chem. Phys. 130 (22), 225102 (2009).

[10]H.S. Hansen and P.H. Hünenberger, J. Comput. Chem. 31, 1-23 (2010).

[1] V. Spiwok, B. Králová and I. Tvaroska, Carbohydr. Res. 345, 530 (2010).

[12] V. Babin and C. Sagui, The Journal of Chemical Physics 132, 104108 (2010).

[13] H.L. Strauss and H.M. Pickett, J. Chem. Phys. 55, 324 (1971).

[14] H.L. Strauss and H.M. Pickett, J. Am. Chem. Soc. 92, 7281 (1970).

[15] V.S.R. Rao, P.K. Qasba, P. Balaji and R. Chandrasekaran, Conformation of Carbohydrates (, , 1998).

[16]S.M. Rings, Eur. J. Biochem. 100, 295-298 (1980).

[17]A. McNaught, Adv. Carbohydr. Chem. Biochem. 52, 43 (1997).

[18] J.E. Kilpatrick, K.S. Pitzer and R. Spitzer, J. Am. Chem. Soc. 64, 2483 (1947).

[19] K. Pitzer and W. Donath, J. Am. Chem. Soc. 81 (13), 3213-3218 (1959).

[20] H. Pickett and H. Strauss, J. Am. Chem. Soc 92, 7281-7290 (1970).

[21]H. Pickett and H. Strauss, J. Chem. Phys. 55, 324 (1971).

[22] J.C.A. Boeyens and D.G. Evans, Acta Cryst. B45, 577 (1989).

[23]L. Bugayevskiy and J. Snyder, Map projections: a reference manual $(,, 1995)$.

[24] A. Laio, A. Rodriguez-Fortea, F. Gervasio, M. Ceccarelli and M. Parrinello, J. Phys. Chem. B 109 (14), 6714-6721 (2005).

[25] M. Mezei, J. Comput. Phys. 68, 237 (1987).

[26] T. Huber, A. Torda and W.F. van Gunsteren, J. Comput.-Aided Mol. Des. 8 (6), 695-708 (1994).

[27] A. Laio and F.L. Gervasio, Rep. Prog. Phys. 71 (12), 126601 (2008).

[28] G. Torrie and J. Valleau, J. Comput. Phys. 23 (2), 187-199 (1977).

[29] V. Babin, C. Roland, T.A. Darden and C. Sagui, J. Chem. Phys. 125, 204909 (2006).

[30]E. Autieri, M. Sega and F. Pederiva 2010 Submitted to J. Chem. Phys.

[31]R. Lins and P. Hünenberger, J. Comput. Chem. 26 (13), 1400 (2005).

[32] H.J.C. Berendsen, J.P.M. Postma, A. DiNola and J.R. Haak, J. Chem. Phys. 81, 3684-3690 (1984).

[33] H.S. Hansen and P.H. Hünenberger, Submitted to J. Comput. Chem. (2010).

[34] M. Parrinello and A. Rahman, J. Appl. Phys. 52, 7182 (1981).

[35] J. Ryckaert, G. Ciccotti and H. Berendsen, J. Comput. Phys. 23 (3), 327-341 (1977). 